Effect of baseline hypocalcaemia on volume of intracerebral haemorrhage in patients presenting within 72 hours from symptom onset

Sallinen, Hanne

2019-08-15

Sallinen , H , Wu , T Y , Meretoja , A , Putaala , J , Tatlisumak , T \& Strbian , D 2019 ,' Effect of baseline hypocalcaemia on volume of intracerebral haemorrhage in patients presenting within 72 hours from symptom onset ' , Journal of the Neurological Sciences, vol. 403 , pp. 24-29 . https://doi.org/10.1016/j.jns.2019.05.033

http://hdl.handle.net/10138/305862

https://doi.org/10.1016/j.jns.2019.05.033

unspecified

publishedVersion

Downloaded from Helda, University of Helsinki institutional repository.

This is an electronic reprint of the original article.

This reprint may differ from the original in pagination and typographic detail.

Please cite the original version. 


\title{
Effect of baseline hypocalcaemia on volume of intracerebral haemorrhage in patients presenting within 72 hours from symptom onset
}

\author{
Hanne Sallinen ${ }^{\mathrm{a}, *}$, Teddy Y. Wu ${ }^{\mathrm{b}}$, Atte Meretoja ${ }^{\mathrm{c}}$, Jukka Putaala ${ }^{\mathrm{a}}$, Turgut Tatlisumak ${ }^{\mathrm{d}}$, \\ Daniel Strbian $^{\mathrm{a}}$ \\ ${ }^{a}$ Department of Neurology, Helsinki University Hospital, University of Helsinki, Helsinki, Finland \\ ${ }^{\mathrm{b}}$ Department of Neurology, Christchurch Hospital, 2 Riccarton Avenue, Christchurch 8011, New Zealand \\ ${ }^{\mathrm{c}}$ Department of Neurology, Helsinki University Hospital, Helsinki, Finland \\ ${ }^{\mathrm{d}}$ Department of Clinical Neuroscience, Department of Clinical Neurology, Institute of Neuroscience and Physiology, Department of Neurology, Sahlgrenska University \\ Hospital, Sahlgrenska Academy at University of Gothenburg, Blå Stråket 7, 3. Floor, Gothenburg 41345, Sweden
}

\section{A R T I C L E I N F O}

\section{Keywords:}

Intracerebral haemorrhage

Haematoma volume

Serum calcium

Stroke outcome

\begin{abstract}
A B S T R A C T
Introduction: Calcium has a pivotal role in haemostasis. We investigated the association of baseline calcium levels with admission intracerebral haemorrhage (ICH) volume.

Methods: This is a retrospective analysis of consecutive ICH patients in an academic hospital between January 2005 and March 2010. Computed tomography (CT) of the brain and serum/plasma ionized calcium had to be taken within $72 \mathrm{~h}$ of symptom onset and within $12 \mathrm{~h}$ of each other in order to fulfil the study criteria. ICH cases related to trauma or tumour as well as sole intraventricular haemorrhages were excluded. Baseline haematoma volumes were calculated using semiautomated planimetry. The hypocalcaemic (Ca-ion $<1.16 \mathrm{mmol} / \mathrm{L}$ ) and normocalcaemic (1.16-1.30 mmol/L) patient groups were compared in univariate analyses. Association between admission hypocalcaemia and haematoma volume was studied using multivariable regression models.

Results: Out of 1013 consecutive patients, 447 fulfilled the study criteria. Hypocalcaemic patients $(n=178$; $39.8 \%$ ) had larger baseline hematoma volumes (median $30.2 \mathrm{~mL}$, IQR $11.4-58.7 \mathrm{~mL}$ ), compared to normocalcaemic patients $(\mathrm{n}=255 ; 57.0 \%$; median $16.8 \mathrm{~mL}$, IQR 7.4-44.2 mL). The median ICH volume among hypercalcaemic patients $(\mathrm{n}=14 ; 3.1 \%$ of included patients) was $6.5 \mathrm{~mL}$ (IQR $3.1-34.6 \mathrm{~mL}$ ). On linear regression, admission hypocalcaemia was independently associated with larger hematoma volumes $(\beta=11.77 ; 95 \% \mathrm{CI}$ 4.66-18.87, $\mathrm{P}=0.01$ ). Patients with larger haematoma volumes had higher mortality.

Conclusion: Hypocalcaemia is associated with larger admission haematoma volumes among ICH patients. Higher mortality among hypocalcaemic patients is very likely mediated through larger ICH volumes.
\end{abstract}

\section{Introduction}

Intracerebral haemorrhage (ICH) results from rupture of an intracerebral artery and causes approximately $10-15 \%$ of all strokes. It is a devastating condition with early mortality rates approaching $40 \%$, and many survivors remaining disabled [1]. There is currently no effective acute intervention for ICH, and treatment is mostly supportive. Known predictors for ICH mortality include older age, lower baseline Glasgow Coma Scale (GCS), higher National Institutes of Health Stroke Scale (NIHSS) score, infratentorial location, ICH and intraventricular haemorrhage (IVH) volumes and their growth, oedema, hyperglycaemia, hydrocephalus, herniation, anticoagulation, and multiple haemorrhages [2-7]. Higher NIHSS score predicts worse functional outcome [8], and greater functional dependence among ICH survivors has been associated with age, GCS, baseline ICH volume, change of ICH volume at $24 \mathrm{~h}$, and IVH [9].

Factors participating in haemostasis and the coagulation cascade have a substantial effect on the prognosis of patients with ICH [10]. The coagulation system includes cellular elements, such as platelets, and the proteins of the coagulation and fibrinolytic pathways. Primary haemostasis involves platelets' adhesion to endothelial proteins, and is followed by secondary haemostasis, i.e. aggregation of platelets and fibrin formation [11]. In the context of an injured vessel wall causing ICH, platelets adhere to the extracellular matrix, become activated, and form a plug to prevent further bleeding. Several platelet activation agonists, such as subendothelial collagen, thromboxane A2, adenosine

\footnotetext{
* Corresponding author at: Department of Neurology, Helsinki University Hospital, Haartmaninkatu 4, PL 340, Helsinki 00029, Finland.

E-mail address: hanne.sallinen@helsinki.fi (H. Sallinen).
} 
diphosphate (ADP) released from the activated platelets, and thrombin generated as a result of the coagulation cascade, trigger signalling pathways that lead to a rise in intracellular $\mathrm{Ca}^{2+}$. The cytosolic calcium derives both from intracellular stores and extracellular sources through the plasma membrane. The elevation of the cytosolic calcium results in further platelet activation and clot formation [12,13]. Calcium is also an important mediator of extracellular coagulation cascade by activating several coagulation factors, such as Factors IX and X to their active forms, and prothrombin to thrombin [14].

Approximately $50 \%$ of the serum calcium is in its active ionized form, and the rest is combined to albumin or other complexes. The main factors regulating the calcium levels are parathyroid hormone, vitamin D, and fibroblast growth factor FGF-23. A number of conditions can lead to hypocalcaemia, including hypoparathyroidism, resistance to parathyroid hormone (PTH), and disorders in vitamin D metabolism and action [15].

Calcium may contribute to ICH volume and outcome through its effect on the coagulation cascade. Studies that have analysed the association between ICH volume and serum calcium levels reported an association between hypocalcaemia and larger ICH volumes, severity of symptoms $(n=273)$ [16], and ICH expansion $(n=2103$; for ICH expansion $n=1393$ ) [17]. Moreover, one study showed elevated calcium levels to be associated with better outcomes $(n=365)$ [18].

Our aim was to examine the association of hypocalcaemia with admission haematoma volume in a large consecutive cohort of nontraumatic ICH patients. The secondary aim was to address the mortality in this cohort.

\section{Methods}

\subsection{Patients}

This is a retrospective analysis of the Helsinki ICH study consisting of 1013 consecutive ICH patients treated at Helsinki University Hospital between January 2005 and March 2010. The methodology and details of this study have been reported elsewhere [10]. Briefly, this cohort includes all consecutive adult ICH patients presenting at the neurology emergency department between January 2005 and March 2010. All underlying causes (including hypertension, anticoagulation, and amyloid angiopathy) of ICH were determined using the SMASH-U classification system $[10,19]$. Patients with ICH from trauma or tumour were excluded. Information on comorbidities, medication and clinical status on admission were obtained from medical records. The severity of the symptoms was assessed using the National Institutes of Health Stroke Scale (NIHSS) and the Glasgow Coma Scale (GCS). Warfarin was the only oral anticoagulant in our cohort. All-cause mortality data was retrieved from national Population Registry Centre last updated in November 2014, which is a reliable source of mortality in Finland, while foreign residents are lost to follow-up.

Patients were included in the present analysis if they had a) ionized plasma/serum calcium taken within $72 \mathrm{~h}$ of symptom onset, b) admission CT of the brain taken within $72 \mathrm{~h}$ of symptom onset, and c) the two taken within $12 \mathrm{~h}$ of each other. When symptom onset time was unknown, we used the last-known-well time as the onset time. We excluded patients with pure intraventricular haemorrhage $(n=5)$. Volume of the ICH was measured by semi-automatic planimetry using Analyze 12.0 software [6,20]. Hypocalcaemia was defined as Caion $<1.16 \mathrm{mmol} / \mathrm{L}$, normocalcaemia Ca-ion $1.16-1.30 \mathrm{mmol} / \mathrm{L}$, and hypercalcaemia as above $1.30 \mathrm{mmol} / \mathrm{L}$ according to our laboratory reference range, and $\mathrm{pH}$ adjusted values were used.

This study has been approved by the institutional review board as an observational registry study with no study-related patient contact. Therefore, no patient consent was required.

\subsection{Statistical analysis}

We expressed categorical variables as counts (\%), and continuous variables as mean (SE) or median (IQR) values after testing for normal distribution. The differences between hypo- and normocalcaemic patients were calculated using the $\chi 2$ test, the $t$-test, or the Mann-Whitney $U$ test as appropriate.

We analysed the association between admission calcium level and baseline ICH volume using multivariable linear regression model. The variables with $\mathrm{P}<0.1$ in the univariate linear regression model (supplemental Table A.2, online only) were included as covariates in the multivariable analysis. Hypocalcaemia was studied as a dichotomous variable; other laboratory test values were not included in the analyses. The time-points for plasma/serum calcium levels were strictly defined as per the study criteria (see above) and all other laboratory test results come from the admission values. Including all laboratory parameters as per the same criteria as were set for calcium would inevitably lead to a major drop in n-number. For these reasons, we included only calcium levels into the analyses.

For the secondary analysis, we used binary logistic regression to study the associations of 90-day mortality and hypocalcaemia. As predictors for mortality are well known, we adjusted our comparison analysis (between hypo- and normocalcaemic patients) for known predictors of mortality (ICH and IVH volume, age, oral anticoagulation, infratentorial location of ICH, and multiple haemorrhages on imaging). NIHSS and GCS are dependent on ICH/IVH volume (collinearity), and were not analysed separately. We tested NIHSS and GCS separately in the same model, and they both had a similar significant association with mortality. The results of the other variables were also similar in those models. Prior medications, infratentorial location, multiple haemorrhages, and hypocalcaemia were included in the models as categorical variables, and age, ICH volume, and IVH volume were included as continuous variables.

As hypercalcaemic patients were few $(n=14)$, we only report their ICH volumes; they were excluded from other analyses. Statistical analyses were made using SPSS v.22 (IBM, Armonk, NY). A two-sided probability $(\mathrm{P})$ value $<0.05$ was considered significant.

\section{Results}

Of the 1013 patients in our database, 447 met the inclusion criteria (Fig. 1).

Supplementary Table A.1 (online only) compares basic characteristics between patients included and excluded in the study. Of included patients, 178 (39.8\%) had hypocalcaemia, and 255 (57.0\%) were normocalcaemic. The median ICH volume among hypercalcaemic patients ( $\mathrm{n}=14$ [3.1\%]) was $6.5 \mathrm{~mL}$ (IQR 3.1-34.6 mL). The comparisons of the baseline characteristics, laboratory values, radiological parameters and outcome parameters between hypocalcaemic and normocalcaemic patients are shown in Table 1.

Compared with normocalcaemic patients, hypocalcaemic patients had larger ICH $(30.2 \mathrm{~mL}$, IQR $11.4-58.7 \mathrm{~mL}$ vs. $16.8 \mathrm{~mL}$, IQR 7.4-44.2 mL; P < 0.001) and IVH volumes $(2.9 \mathrm{~mL}$, IQR $0.0-22.5 \mathrm{~mL}$ vs. $0.0 \mathrm{~mL}$, IQR $0.0-8.7 \mathrm{~mL}$; $>0.001$ ). The hypocalcaemic patients were more often male $(66.9 \%$ vs. $56.9 \%$; $\mathrm{P}=0.04)$, less frequently dyslipidaemic $(13.3 \%$ vs. $21.7 \%$; $\mathrm{P}=0.03)$, had higher admission NIHSS scores (19.0, IQR 10.0-34.0 vs. 12.0, IQR 6.0-21.0; P < 0.001) and lower GCS scores (10.0, IQR 4.0-14.0 vs. 14.0, IQR 10.0-15.0; $\mathrm{P}<0.001)$, had higher white cell count (9.7, IQR 7.2-12.0 vs. 8.0, IQR 6.4-10.4; $\mathrm{P}<0.001$ ), C-reactive protein (CRP) (5.0, IQR 3.0-7.0 vs. 4.0, IQR 3.0-6.0; $\mathrm{P}=0.017$ ), and glucose on admission or in ambulance (8.4, IQR 6.9-10.8 vs. 7.5, IQR 6.3-9.5; P < 0.001), and lower potassium (3.7, IQR 3.3-4.0 vs. 3.8, IQR 3.5-4.1; P = 0.018), and sodium (137.0, IQR $135.0-139.0$ vs. 138.0 , IQR 136.0-140.0, P = 0.046) values along with lower creatinine values (63.0, IQR 54.0-82.5 vs. 69.0, IQR 58.0-85.8; $\mathrm{P}=0.040$ ). Hydrocephalus was more common 


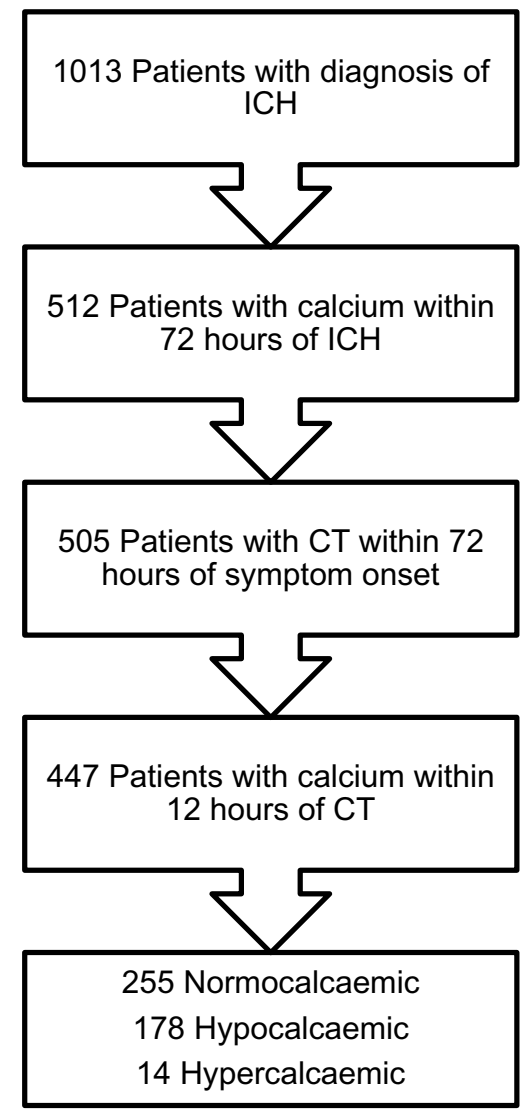

Fig. 1. Cohort selection flow chart.

Abbreviations: ICH: intracerebral haemorrhage; CT: computed tomography

among hypocalcaemic patients (30.9\% vs. $12.2 \%$; P $<0.001)$, but was not included in the analyses or tables. There was no difference between the rate of ventricular drainage in the two groups (hypocalcaemic $5.1 \%$ vs. normocalcaemic $3.5 \%$; $\mathrm{P}=0.43$ ).

The univariate analyses of parameters related to ICH volume are depicted in supplementary Table A.2 (online only). In multivariable linear regression model, we found that admission hypocalcaemia was strongly independently associated with larger ICH volumes $(\beta=11.77$, $95 \%$ CI 4.66-18.87, $\mathrm{P}=0.001$ ). The only other variable independently associated with admission ICH volume in our cohort was older age (age per year $\beta=0.45,95 \%$ CI $0.16-0.74, P=0.002$ ), Table 2. Multivariable linear regression analysis using calcium as continuous variable also demonstrated an inverse relationship $(\beta=-87.72,95 \%$ CI -138.58- $-36.86, P=0.001$ ) between increasing serum calcium level and baseline haematoma volume shown in supplementary Table A.3 (online only).

In unadjusted analysis, three-month mortality was significantly higher among hypocalcaemic patients than in normocalcaemic patients (53.1\% vs. $34.5 \%$; $\mathrm{P}<0.001)$. The univariate analyses of factors related to 3-month mortality are listed in supplementary table A.4 (online only). In the multivariable logistic regression model hypocalcaemia was not associated with mortality (OR $0.69,95 \%$ CI 3.58-12.96, P $=0.13$ ) after adjusting for known predictors of mortality including haematoma volume (Table 3). The variables independently associated with mortality were older age (age per year OR 1.04, 95\% CI 1.02-1.06, $\mathrm{P}<0.001$ ), larger ICH (OR 1.03, 95\% CI 1.02-1.04, P < 0.001) and IVH (OR 1.04, 95\% CI 1.03-1.06, P < 0.001) volumes, and infratentorial location of ICH (OR 6.81, 95\% CI 3.58-12.96, P < 0.001), Table 3.

\section{Discussion}

We have demonstrated that a significant portion of acute ICH patients $(\sim 40 \%)$ were hypocalcaemic, and that a clear association existed between hypocalcaemia and larger admission ICH volumes. The hypocalcaemic patients had higher 90-day mortality, which was likely mediated through larger ICH volumes.

Hypocalcaemia has been shown to be common among critically ill patients, and to associate with higher mortality [21]. The proposed causes of hypocalcaemia in critically ill patients include catecholaminemediated translocation of plasma calcium into tissues and increased faecal and/or urinary calcium excretion, and hyperadraenergic state has been shown to be associated with calcium derangement [21]. Thus, hypocalcaemia may be a reflector of poor outcome in critically ill patients. However, in our cohort, hypocalcaemia was not independently associated with mortality. This was not unexpected because the haematoma volume was included in the same model. Hypocalcaemia was strongly and independently associated with larger ICH volume, which belongs to most important predictors of mortality among ICH patients.

Previous studies have reported an association between hypocalcaemia with larger ICH volumes [16,17]. In a cohort of 273 patients, the patients were divided in quartiles according to their calcium levels. The median ICH volume in the lowest serum calcium quartile ( $\leq$ $2.25 \mathrm{mmol} / \mathrm{L})$ was significantly higher $(\mathrm{P}=0.005)$, whereas in all other groups the ICH volumes were similar [16]. There was no significant association between hypocalcaemia and mortality after adjustment with NIHSS and hematoma volume [16]. Morotti et al. reported larger baseline ICH volume in hypocalcaemic patients (37 mL (IQR 15-72 mL) when compared with nomocalcaemic patients (16 mL (IQR 6-44 mL), $\mathrm{P}<0.001$ ) in $2103 \mathrm{ICH}$ patients but the authors did not report outcome analysis [17]. In another study of 365 ICH patients the ICH volumes in different quartiles of serum calcium did not significantly differ between hypo-, normo- and hypercalcaemic patients, but hypercalcaemic (serum calcium $\geq 2.32 \mathrm{mmol} / \mathrm{L}$ ) patients had better outcome [18]. Finally, Liotta et al. reported in 290 ICH patients that lower serum magnesium level on admission was associated with larger haematoma volumes, which suggests that magnesium levels may play a pathophysiologic role in the haemostasis of ICH patients [22]. In that study, admission calcium was not significantly associated with ICH volume or expansion.

In our study, the proportion of hypocalcaemic patients (39.8\%) was higher compared to previously published studies (10.9\% [17], $22.7 \%$ in the lowest quartile [16], and $24.7 \%$ in the lowest quartile [18]). In those studies, however, total serum calcium levels were used, or, additionally, ionized calcium was used as a continuous variable [17]. Thus, the proportion of hypocalcaemic patients is not fully comparable with our data.

The effect of calcium on coagulation and association with blood pressure have been suggested as potential mechanisms for larger ICH volumes in earlier studies. However, in an earlier study, the hypocalcaemic patients did not have significantly higher blood pressure compared to normocalcaemic patients [17]. In our study, the admission blood pressures did not differ significantly among hypo- and normocalcaemic patients. It has also been suggested that hypocalcaemia might reflect poor liver function, as one of the studies found that liver disease was more common among hypocalcaemic ICH patients [16]. However, in our cohort, liver disease was not significantly more common among hypocalcaemic patients. In our study, age, ICH and IVH volumes and infratentorial location were independent predictors of mortality, which is in line with earlier studies [2]. Apart from dyslipidaemia, the medical history was similar among hypo- and normocalcaemic patients (supplementary Table A.1). In a study on the effect of hypocalcaemia on coagulation, calcium had a concentration-dependent association with in vitro clot strength after adjusting for other coagulation abnormalities [23]. In bleeding trauma patients receiving massive transfusions, ionized calcium concentrations had an inverse 
Table 1

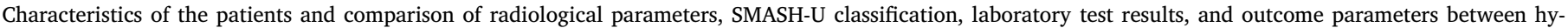
pocalcemic and normocalcemic patients.

\begin{tabular}{|c|c|c|c|c|}
\hline Variable & $\begin{array}{l}\text { Hypocalcemic patients } \\
(\mathrm{n}=178)\end{array}$ & Normocalcemic patients $(\mathrm{n}=255)$ & $P$ value & $\begin{array}{l}\text { Missing values hypocalcemia/ } \\
\text { normocalcemia }\end{array}$ \\
\hline Age in years & $62.0(57-71)$ & $65.0(57-76)$ & 0.12 & $0 / 0$ \\
\hline Male & $119(66.9)$ & $145(56.9)$ & 0.04 & $0 / 0$ \\
\hline Pre-ICH mRS 3-5 & $8(4.5)$ & $13(5.1)$ & 0.77 & $0 / 0$ \\
\hline \multicolumn{5}{|l|}{ Comorbidities } \\
\hline Hypertension & $161(63.1)$ & $109(61.2)$ & 0.69 & $0 / 0$ \\
\hline Diabetes & $28(15.7)$ & $39(15.3)$ & 0.9 & $0 / 0$ \\
\hline Dyslipidaemia & $23(13.3)$ & $55(21.7)$ & 0.03 & $5 / 1$ \\
\hline History of ischemic stroke & $21(11.8)$ & $27(10.6)$ & 0.69 & $0 / 0$ \\
\hline History of ICH & $8(4.5)$ & $8(3.1)$ & 0.46 & $0 / 0$ \\
\hline Liver disease & $6(3.5)$ & $8(3.1)$ & 0.85 & $6 / 1$ \\
\hline Coronary artery disease & $19(11.0)$ & $26(10.3)$ & 0.80 & $6 / 2$ \\
\hline Atrial fibrillation & $19(10.7)$ & 33 (12.9) & 0.48 & $0 / 0$ \\
\hline Epilepsy & $5(2.9)$ & $10(3.9)$ & 0.56 & $5 / 1$ \\
\hline \multicolumn{5}{|l|}{ Medications prior to ICH } \\
\hline Antiplatelets & $41(23.0)$ & $56(22.0)$ & 0.81 & $0 / 1$ \\
\hline Oral anticoagulants & $18(10.1)$ & $34(13.3)$ & 0.31 & $0 / 0$ \\
\hline Statins & $22(12.9)$ & $50(19.9)$ & 0.059 & $7 / 4$ \\
\hline Antihypertensives & $75(42.1)$ & $123(48.2)$ & 0.21 & $0 / 0$ \\
\hline \multicolumn{5}{|l|}{ Clinical admission parameters } \\
\hline NIHSS & $19.0(10.0-34.0)$ & $12.0(6.0-21.0)$ & $<0.001$ & $0 / 0$ \\
\hline GCS & $10.0(4.0-14.0)$ & $14.0(10.0-15.0)$ & $<0.001$ & $0 / 0$ \\
\hline Systolic blood pressure, mmHg & $175.6(2.91)$ & $172.2(2.26)$ & 0.35 & $17 / 28$ \\
\hline Diastolic blood pressure, $\mathrm{mmHg}$ & $94.6(1.93)$ & $91.7(1.38)$ & 0.17 & $17 / 31$ \\
\hline Time from ICH onset to baseline CT, hours & $3.5(1.6-9.8)$ & $2.1(1.4-4.8)$ & 0.002 & $0 / 0$ \\
\hline \multicolumn{5}{|l|}{ Radiological parameters on admission CT } \\
\hline $\mathrm{ICH}$ volume, $\mathrm{mL}$ & $30.2(11.4-58.7)$ & $16.8(7.4-44.2)$ & $<0.001$ & $0 / 0$ \\
\hline Oedema volume, $\mathrm{mL}$ & $18.0(6.6-37.2)$ & $11.6(5.6-30.4)$ & 0.11 & $0 / 0$ \\
\hline IVH volume, $\mathrm{mL}$ & $2.9(0.0-22.5)$ & $0.0(0.0-8.7)$ & $<0.001$ & $0 / 0$ \\
\hline ICH location & & & 0.14 & $0 / 0$ \\
\hline Lobar & $54(30.3)$ & $92(36.1)$ & & \\
\hline Deep & $91(51.1)$ & $132(51.8)$ & & \\
\hline Infratentorial & $33(18.5)$ & $31(12.2)$ & & \\
\hline Multiple haemorrhages & $9(5.1)$ & $10(3.9)$ & 0.571 & $0 / 0$ \\
\hline SMASH-U classification & & & 0.21 & $0 / 0$ \\
\hline Amyloid angiopathy & $23(12.9)$ & $48(18.8)$ & & \\
\hline Hypertension & $74(41.6)$ & $92(36.1)$ & & \\
\hline Medication & $22(12.4)$ & $36(14.1)$ & & \\
\hline Other disease & $11(6.2)$ & $12(4.7)$ & & \\
\hline Structural lesion & $4(2.2)$ & $14(5.5)$ & & \\
\hline Undetermined & $44(24.7)$ & $53(20.8)$ & & \\
\hline \multicolumn{5}{|l|}{ Admission laboratory results } \\
\hline Haemoglobin, g/L & $138.1(1.4)$ & $138.5(1.1)$ & 0.93 & $3 / 3$ \\
\hline White cell count, $10^{9} / \mathrm{L}$ & $9.7(7.2-12.0)$ & $8.0(6.4-10.4)$ & $<0.001$ & $2 / 3$ \\
\hline Platelet count, $10^{9} / \mathrm{L}$ & $211.1(6.2)$ & $213.6(4.1)$ & 0.53 & $6 / 4$ \\
\hline $\mathrm{CRP}, \mathrm{mg} / \mathrm{dL}$ & $5.0(3.0-7.0)$ & $4.0(3.0-6.0)$ & 0.017 & $2 / 2$ \\
\hline INR & $1.0(1.0-1.2)$ & $1.0(0.9-1.2)$ & 0.500 & $33 / 49$ \\
\hline $\begin{array}{l}\text { Glucose on admission or in ambulance, mmol/ } \\
\quad \mathrm{L}\end{array}$ & $8.4(6.9-10.8)$ & $7.5(6.3-9.5)$ & $<0.001$ & $1 / 1$ \\
\hline Potassium, mmol/L & $3.7(3.3-4.0)$ & $3.8(3.5-4.1)$ & 0.018 & $6 / 5$ \\
\hline Sodium, mmol/L & $137.0(135.0-139.0)$ & $138.0(136.0-140.0)$ & 0.046 & $5 / 5$ \\
\hline Creatinine, $\mu \mathrm{mol} / \mathrm{L}$ & $63.0(54.0-82.5)$ & $69.0(58.0-85.8)$ & 0.040 & $9 / 7$ \\
\hline Any neurosurgery & $28(15.7)$ & $31(12.2)$ & 0.29 & $0 / 0$ \\
\hline 90-d-Mortality & $93(53.1)$ & $87(34.5)$ & $<0.001$ & $3 / 3$ \\
\hline
\end{tabular}

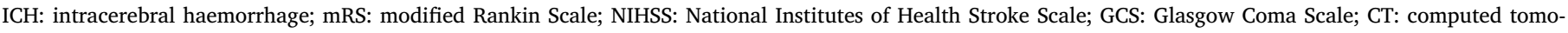
graphy; IVH: intraventricular haemorrhage; SMASH-U classification: etiologic classification of ICH; CRP: C-reactive protein.

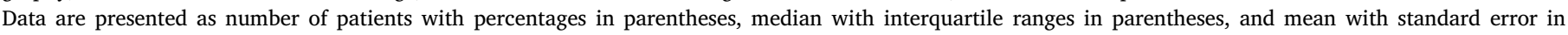
parentheses.

Bold is $\mathrm{P}$ value $<0.05$.

concentration-dependent relationship with mortality [24], and hypocalcaemia has been thought to be one of the factors altering haemostasis, and contributing to morbidity and mortality of bleeding patients in that setting [25].

It has been shown in animal models that ischaemia in neurons leads to prolonged calcium influx into the cells from extracellular sources [26]. In an experimental model of ICH in cats, there was a depletion of calcium ions in the extracellular space, and the calcium levels did not return to the baseline level. The ionic movement also took place in cells not directly damaged [27], suggesting a relationship between endothelial dysfunction and hypocalcaemia. As the prior-ICH calcium levels are not known, we cannot rule out the neuronal damage itself having an effect on blood calcium levels after the ictus, or that hypocalcaemic patients having larger ICH volumes would have a larger amount of calcium-ions being drawn from the blood to the larger hematoma and the surrounding penumbra. Also, as factors related to 
Table 2

Multivariable linear regression analysis for ICH volume predictors.

\begin{tabular}{|c|c|c|c|c|}
\hline & $\beta(95 \% \mathrm{CI})$ & SE & $P$ value & Missing data \\
\hline Hypocalcaemia & $11.77(4.66-18.87)$ & 3.62 & 0.001 & 0 \\
\hline Age (per year) & $0.45(0.16-0.74)$ & 0.15 & 0.002 & 0 \\
\hline Use of statin prior to ICH & $9.55(-0.80-19.90)$ & 5.26 & 0.070 & 11 \\
\hline Antiplatelet medication prior to ICH & $3.93(-5.46-13.31)$ & 4.77 & 0.41 & 1 \\
\hline Use of oral anticoagulant (warfarin) prior to ICH & $8.79(-2.53-20.10)$ & 5.76 & 0.13 & 0 \\
\hline Antihypertensive medication prior to ICH & $3.41(-4.51-11.33)$ & 4.03 & 0.40 & 0 \\
\hline
\end{tabular}

CI: confidence interval; SE: standard error; ICH: intracerebral haemorrhage.

Bold is $\mathrm{P}$ value $<0.05$.

Table 3

Multivariable analysis for predictors of mortality at 90 days $(n=427)$.

\begin{tabular}{|c|c|c|c|c|}
\hline & $\mathrm{OR}$ & $\begin{array}{l}95 \% \text { confidence } \\
\text { interval }\end{array}$ & $P$ value & Missing data \\
\hline Age, per year & 1.04 & $1.02-1.06$ & $<0.001$ & 0 \\
\hline Volume of ICH, mL & 1.03 & $1.02-1.04$ & $<0.001$ & 0 \\
\hline Volume of IVH, mL & 1.04 & $1.03-1.06$ & $<0.001$ & 0 \\
\hline $\begin{array}{l}\text { Infratentorial location of } \\
\text { ICH }\end{array}$ & 6.81 & $3.58-12.96$ & $<0.001$ & 0 \\
\hline Hypocalcaemia & 0.69 & $0.42-1.12$ & 0.13 & 0 \\
\hline $\begin{array}{l}\text { Anticoagulant medication } \\
\text { (warfarin) prior to ICH }\end{array}$ & 1.08 & $0.49-2.39$ & 0.85 & 0 \\
\hline Multiple ICHs on imaging & 1.72 & $0.59-5.04)$ & 0.32 & 0 \\
\hline
\end{tabular}

OR: odds ratio; ICH: intracerebral haemorrhage; IVH, intraventricular haemorrhage.

Bold is $P$ value $<0.05$.

possible pre-ICH hypocalcaemia, such as vitamin D, and parathyroid hormone levels, are not known, we cannot rule out these underlying states itself affecting the haematoma volume.

The association between hypocalcaemia and ICH volume is likely mediated through the negative influence of hypocalcaemia on coagulation. The higher mortality rates seen in hypocalcaemic patients is likely to be accounted for by larger ICH volume which is one of the most powerful predictors of mortality [2].

The strengths of our study are utilization of planimetry methods, and consecutive character of the cohort. We used the ionized calcium level, which reflects better the physiologically active calcium [28]. For obtaining the most relevant values, we limited the timing of the laboratory test to $12 \mathrm{~h}$ from the admission CT and both brain CT and ionized calcium measurements to $72 \mathrm{~h}$ of symptom onset. The majority of patients were admitted within the first hours after the ictus (Supplemental Fig. A.1).

The limitations of our study stem from its retrospective nature. We also had a large proportion of patients with missing early calcium values. The possible selection bias for analysing calcium for certain patients could not be out ruled. The timing of the CT and blood samples in relation to symptom onset varied, limiting our ability to evaluate a potential relationship between calcium levels and ICH growth. As the median time from ICH onset to CT was longer (median $3.5 \mathrm{~h}$, IQR $1.6-9.8$ vs. $2.1 \mathrm{~h}$, IQR $1.4-4.8 ; \mathrm{p}=0.002$ ) in the hypocalcaemic group, we cannot rule out the time itself having some effect on ICH volume; however, in linear multivariate analysis, time was not an independent predictor on ICH volume. Due to the acute nature of the illness, many patients are expected to have received intravenous fluids already before arriving to hospital, before laboratory testing. However, the amount of such fluid intake could not be evaluated retrospectively, which is the main reason for applying our inclusion criteria, i.e. the maximum time delayed allowed between ICH onset and laboratory testing. Finally we were unable to examine the association between hypocalcaemia and functional recovery as follow up was not routine at our institution during the study period. Mortality however is a robust endpoint and information on mortality was derived from a reliable source.
In future, prospective clinical studies could be planned to take into consideration and overcome some of the limitations of our study. The blood samples could be collected as early as possible, and preferably before intravenous fluid administration. To find out the possible causes for hypocalcaemia, more extensive laboratory testing, including vitamin D, and parathyroid hormone could be done, as well as repeated sampling of calcium to find out possible changes with time in the face of acute ICH. Interesting topic would be to investigate, if correction of hypocalcaemia in ICH patients affects their prognosis.

\section{Conclusion}

Admission hypocalcaemia among ICH patients was associated with larger ICH volumes, and hypocalcaemic patients had higher 90-day mortality than normocalcaemic patients ( $53.1 \%$ vs. $34.5 \%)$, which was driven by larger ICH volumes in hypocalcaemic patients. Larger prospective studies are needed to further examine the relationship between serum calcium and ICH volume.

\section{Declarations of interests}

TT reports grants from Helsinki University Central Hospital, grants from University of Gothenburg, grants from Sahlgrenska University Hospital, grants from Sigrid Juselius Foundation, during the conduct of the study; grants and personal fees from Boehringer-Ingelheim, personal fees from Bayer, personal fees from Pfizer, grants from BrainsGate, grants from Bayer, grants from Pfizer, personal fees from Lumosa Pharm, outside the submitted work; In addition, Dr. Tatlisumak has a patent Use of a mast cell activation or degranulation blocking agent in the manufacture of a medicament for the treatment of a patient subjected to thrombolyses. Patent no: US8163734. Filed: February 13, 2004. Issued: April 24, 2012. HS, TW, AM, JP and DS report no conflicts of interests.

\section{Acknowledgements}

None.

\section{Funding}

This work was supported by the Finnish Governmental subsidiary grant (VTR), and grants from Maire Taponen Foundation and The Finnish Medical Foundation.

\section{Appendix A. Supplementary data}

Supplementary data to this article can be found online at https:// doi.org/10.1016/j.jns.2019.05.033.

\section{References}

[1] C.J. van Asch, M.J. Luitse, G.J. Rinkel, I. van der Tweel, A. Algra, C.J. Klijn, Incidence, case fatality, and functional outcome of intracerebral haemorrhage over 
time, according to age, sex, and ethnic origin: a systematic review and meta-analysis, Lancet Neurol. 9 (2010) 167-176.

[2] J. Hemphill, D. Bonovich, L. Besmertis, G. Manley, S. Johnston, The ICH score: a simple, reliable grading scale for intracerebral hemorrhage, Stroke. 32 (2001) 891-897.

[3] R.-J. Koivunen, J. Satopää, E. Haapaniemi, D. Strbian, A. Meretoja, S. Mustanoja, et al., Predictors of early mortality in young adults after intracerebral hemorrhage, Stroke. 45 (2014) 2454-2456.

[4] S. Mustanoja, J. Satopää, A. Meretoja, J. Putaala, D. Strbian, S. Curtze, et al., Extent of secondary intraventricular hemorrhage is an independent predictor of outcomes in intracerebral hemorrhage: data from the Helsinki ICH study, Int. J. Stroke 10 (2014) 576-581.

[5] J.B. Kuramatsu, S.T. Gerner, P.D. Schellinger, J. Glahn, M. Endres, J. Sobesky, et al., Anticoagulant reversal, blood pressure levels, and anticoagulant resumption in patients with anticoagulation-related intracerebral hemorrhage, Jama. 313 (2015) 824-836.

[6] T.Y. Wu, G. Sharma, D. Strbian, J. Putaala, P. smond, T. Tatlisumak, et al., Natural history of perihematomal edema and impact on outcome after intracerebral hemorrhage, Stroke. 48 (2017) 873-879.

[7] T.Y. Wu, N. Yassi, D.G. Shah, M. Ma, G. Sharma, J. Putaala, et al., Simultaneous multiple intracerebral hemorrhages (SMICH), Stroke. 48 (2017) 581-586.

[8] C. Finocchi, M. Balestrino, L. Malfatto, G. Mancardi, C. Serrati, C. Gandolfo, National Institutes of Health Stroke Scale in patients with primary intracerebral hemorrhage, Neurol. Sci. 39 (2018) 1751-1755.

[9] J. Pinho, A. Costa, J. Araújo, J. Amorim, C. Ferreira, Intracerebral hemorrhage outcome: a comprehensive update, J. Neurol. Sci. 398 (2019) 54-66.

[10] A. Meretoja, D. Strbian, J. Putaala, S. Curtze, E. Haapaniemi, S. Mustanoja, et al. SMASH-U: a proposal for etiologic classification of intracerebral hemorrhage, Stroke. 43 (2012) 2592-2597.

[11] H. Spronk, J.W. Govers-Riemslag, H. ten Cate, The blood coagulation system as a molecular machine, Bioessays. 25 (2003) 1220-1228.

[12] D. Varga-Szabo, A. Braun, B. Nieswandt, Calcium signaling in platelets, J. Thromb. Haemost. 7 (2009) 1057-1066.

[13] T.J. Stalker, D.K. Newman, P. Ma, K.M. Wannemacher, L.F. Brass, Platelet Signaling, http://eutils.ncbi.nlm.nih.gov/entrez/eutils/elink.fcgi?dbfrom = pubmed\&id $=22918727 \&$ retmode $=$ ref\&cmd $=$ prlinks $($ accessed 05/10/2017).

[14] A.C. Guyton, J.E. Hall, Guyton and Hall Textbook of Medical Physiology, 12 ed., Elsevier Saunders, Philadelphia, 2011.

[15] L.J. Groot, P. Beck-Peccoz, G. Chrousos, K. Dungan, A. Grossman, J.M. Hershman, et al., Hypocalcemia: Diagnosis and Treatment, https://www.ncbi.nlm.nih.gov/ books/NBK279022/, Accessed date: 6 April 2018

[16] Y. Inoue, F. Miyashita, K. Toyoda, K. Minematsu, Low serum calcium levels contribute to larger hematoma volume in acute intracerebral hemorrhage, Stroke. 44 (2013) 2004-2006.

[17] A. Morotti, A. Charidimou, C.-L. Phuah, M.J. Jessel, K. Schwab, A.M. Ayres, et al., Association between serum calcium level and extent of bleeding in patients with Intracerebral Hemorrhage, Jama Neurol. 73 (2016) 1285-1290.

[18] S. You, Q. Han, J. Xu, C. Zhong, Y. Zhang, H. Liu, et al., Serum calcium and phosphate levels and short- and long-term outcomes in acute intracerebral hemorrhage patients, J. Stroke Cerebrovasc. Dis. 25 (2016) 914-920.

[19] K. Rannikmäe, R. Woodfield, C.S. Anderson, A. Charidimou, P. Chiewvit, S.M. Greenberg, et al., Reliability of intracerebral hemorrhage classification systems: a systematic review, Int. J. Stroke 11 (2016) 626-636.

[20] T.Y. Wu, O. Sobowale, R. Hurford, G. Sharma, S. Christensen, N. Yassi, et al., Software output from semi-automated planimetry can underestimate intracerebral haemorrhage and peri-haematomal oedema volumes by up to 41 , Neuroradiology. 58 (2016) 867-876.

[21] Z. Zhang, X. Xu, H. Ni, H. Deng, Predictive value of ionized calcium in critically ill patients: an analysis of a large clinical database MIMIC II, PLoS One 9 (2014) e95204.

[22] E.M. Liotta, S. Prabhakaran, R.S. Sangha, R.A. Bush, A.E. Long, S.A. Trevick, et al., Magnesium, hemostasis, and outcomes in patients with intracerebral hemorrhage, Neurology. 89 (2017) 813-819.

[23] K. Ho, C. Yip, Concentration-dependent effect of hypocalcaemia on in vitro clot strength in patients at risk of bleeding: a retrospective cohort study, Transfus. Med. 26 (2016) 57-62.

[24] K. Ho, A. Leonard, Concentration-dependent effect of hypocalcaemia on mortality of patients with critical bleeding requiring massive transfusion: a cohort study, Anaesth. Intensive Care 39 (2011) 46-54.

[25] D.E. Robertis, S. Kozek-Langenecker, R. Tufano, G. Romano, O. Piazza, Z.G. Marinosci, Coagulopathy induced by acidosis, hypothermia and hypocalcaemia in severe bleeding, Minerva Anestesiol. 81 (2014) 65-75.

[26] T. Kristian, B. Siesjo, Calcium in ischemic cell death, Stroke. 29 (1998) 705-718.

[27] O. Hubschmann, D. Nathanson, The role of calcium and cellular membrane dysfunction in experimental trauma and subarachnoid hemorrhage, J. Neurosurg. 62 (1985) 698-703.

[28] L.M. Calvi, D.A. Bushinsky, When is it appropriate to order an ionized calcium? J. Am. Soc. Nephrol. 19 (2008) 1257-1260. 